\title{
Assessment of Esophageal Motor Disorders Using High-resolution Manometry in Esophageal Dysphagia With Normal Endoscopy
}

\author{
Dan Wang, ${ }^{*}$ Xiu Wang, Yao Yu, Xiaowen Xu, Jing Wang, Yuting Jia, and Hong $\mathrm{Xu}^{*}$ \\ Department of Gastroenterology, the First Hospital of Jilin University, China
}

\section{Background/Aims}

The distribution and esophageal motor characteristics of Chinese patients with esophageal dysphagia who exhibit no structural abnormalities on esophagogastroduodenoscopy remain unclear. Our aim is to assess the esophageal motor patterns using highresolution manometry (HRM) and classify them according to the Chicago classification version 3.0 (CC v3.0). Furthermore, we compared the CC v3.0 and the previous version 2.0 (CC v2.0) for diagnosis of motor disorders.

\section{Methods}

Two hundred thirty-six (mean age $48.4 \pm 12.2$ years, 61.9\% female) patients with esophageal dysphagia were included for analysis of motor function using HRM. All participants were administered a questionnaire to determine Eckardt scores before HRM.

\section{Results}

According to the CC v3.0, 57 (24.2\%) patients showed evidence of esophagogastric junction outflow obstruction and were classified as Group 1. Eighteen (7.6\%) patients with major disorders of peristalsis were classified as Group 2. Minor disorders of peristalsis (Group 3) were much more frequent (129 [54.7\%] patients). Thirty-two (13.6\%) patients had normal esophageal manometry were classified as Group 4. All patients with abnormal pH or pH impedance monitoring $(n=44)$ had minor motor disorders (ineffective esophageal motility [IEM] $=34$, fragmented peristalsis $=10$ ). Based on motor category, the Eckardt score was $4.7 \pm 0.1$ in Group 1, $4.5 \pm 0.3$ in Group 2, $3.5 \pm 0.1$ in Group 3, and $3.9 \pm 0.1$ in Group 4.

\section{Conclusions}

IEM was the most common esophageal motor disorder in patients with esophageal dysphagia who showed no structural abnormality on endoscopy. While a high Eckardt score suggests outflow obstruction or a major motor disorder, a low score suggests IEM.

\section{(J Neurogastroenterol Motil 2019;25:61-67)}

\section{Key Words}

Dysphagia; Endoscopy, digestive system; Esophageal motility disorder; Manometry

Received: March 3, 2018 Revised: June 12, 2018 Accepted: October 9, 2018

(5) This is an Open Access article distributed under the terms of the Creative Commons Attribution Non-Commercial License (http://creativecommons. org/licenses/by-nc/4.0) which permits unrestricted non-commercial use, distribution, and reproduction in any medium, provided the original work is properly cited.

${ }^{*}$ Correspondence: Dan Wang and Hong Xu are equally responsible for this study. Dan Wang, MD Department of Gastroenterology, The First Hospital of JiLin University, No. 71, XinMin Street, ChangChun 130021, China Tel: +86-43188782821, Fax: +86-43188786166, E-mail: doctorwd@163.com Hong $\mathrm{Xu}, \mathrm{MD}$

Department of Gastroenterology, The First Hospital of Jilin University, No. 71, XinMin Street, ChangChun 130021, China Tel: +86-13756661648, Fax: +86-43188786166, E-mail: chxuhong@163.com 


\section{Introduction}

Esophageal dysphagia refers to the sensation of difficulty in swallowing solids and/or liquids in the esophagus. ${ }^{1}$ It is a common symptom that incorporates a wide range of etiologies and is generally classified as obstructive or non-obstructive dysphagia (NOD). NOD was earlier defined as difficulty in swallowing in the absence of any anatomical evidence of stenosis in the esophagus, as determined by radiological and/or endoscopic evaluation. ${ }^{2}$ More recently, the term NOD has been used to connote absence of any motility disorder that may result in obstruction of bolus transit. ${ }^{3}$ The ambiguous definition is a cause of confusion during clinical evaluation of patients with esophageal dysphagia, especially while determining the likely etiology. Therefore it is important to find an appropriate and standardized method to assess esophageal dysphagia. Generally, esophagogastroduodenoscopy (EGD) is the diagnostic modality of choice to rule out obstructive dysphagia, particularly of mechanical etiology. Subsequently, distinguishing the motor pattern of esophageal motor disorders is a key step in the workup of patients with normal EGD and biopsies. Methodologies for characterization of motor disorders have lacked standardization until the application of high-resolution manometry (HRM).

HRM represents a revolutionary advance over conventional manometry and has become the standard method for diagnosis of esophageal motor disorders. ${ }^{4}$ The Chicago classification version 3.0 (CC v3.0) provides the current updated criteria for diagnosis and classification of esophageal motor disorders. However, the distribution characteristics and the classification of motor disorders in patients with esophageal dysphagia who show no evidence of structural abnormality on upper endoscopy (EGD) are not clear in China. There is an urgent need to classify esophageal motor disorders correctly in order to guide treatment and assess efficacy. The aim of this study is to classify motor disorders using HRM in Chinese patients with esophageal dysphagia who have normal EGD. We further analyzed the association between the severity of symptoms and the categories of esophageal motor disorders.

\section{Materials and Methods}

\section{Subjects}

Adult patients $(\geq 18$ years $)(n=256)$ who had esophageal dysphagia as the predominant symptom and had normal esophageal appearance on EGD were retrospectively identified at the First
Hospital of JiLin University during a 4-year period between Jan 2011 and Jan 2015. An apparently normal esophageal appearance in EGD implied absence of dilated esophagus and/or food residue, elevated lower esophageal sphincter (LES) resistance, or significant characteristics of hiatus hernia $(\mathrm{HH})$. All subjects completed a standard esophageal HRM using the Given system (Given Imaging/Sierra Scientific, Los Angeles, CA, USA).

Exclusion criteria were: individuals with $\mathrm{HH}$ ( $\geq 1 \mathrm{~cm}$ separation between the LES and crural diaphragm), or secondary esophageal motor disorders such as desmosis, or systemic sclerosis (scleroderma). Patients with suspected non-erosive reflux disease (NERD) completed 24-hour $\mathrm{pH}$ monitoring or impendence monitoring.

Informed consent was obtained from each subject as routine practice prior to conducting esophageal HRM. The study protocol was approved by the Human Ethics Committee of the First Hospital of JiLin University, China (IRB No. 2014-311).

\section{Esophageal High-resolution Manometry Criteria}

After an overnight fast, HRM was performed using a 36-channel solid-state catheter system with high-fidelity circumferential sensors at 1-cm intervals (Given Imaging/Sierra Scientific). After calibration, the catheter was passed through an anesthetized nasal canal. A 20-second swallow-free period was first obtained after the subject remained in a resting state in the recumbent position (landmark period), from which basal LES pressures were obtained. Ten swallows were recorded using 4-5 $\mathrm{mL}$ of water at ambient temperature spaced $>20$ seconds apart. Studies were acquired and analyzed using dedicated computerized HRM acquisition, display and analysis systems (ManoView; Given Imaging/Sierra Scientific). ${ }^{5}$ Standard analysis of the motor pattern was performed using the Chicago classification version $3.0(\mathrm{CC} \text { v } 3.0)^{6}$ and version $2.0(\mathrm{CC}$ v2.0).

According to the hierarchical algorithm for the interpretation of HRM studies with CC v3.0, ${ }^{6}$ esophageal motor disorders were divided into 4 main groups: Group 1, esophagogastric junction outflow obstruction (EGJOO), including achalasia and EGJOO; Group 2, major disorders of peristalsis including absence of contractility, distal esophageal spasm (DES), and hypercontractile esophagus (jackhammer); Group 3, minor disorders of peristalsis including ineffective esophageal motility (IEM) and fragmented peristalsis; and Group 4, normal esophageal manometry with normal esophageal HRM study (Fig. 1).

\section{Questionnaire}

Patients who met the inclusion criteria were administered a Chinese version of the questionnaire for calculation of the Eckardt 


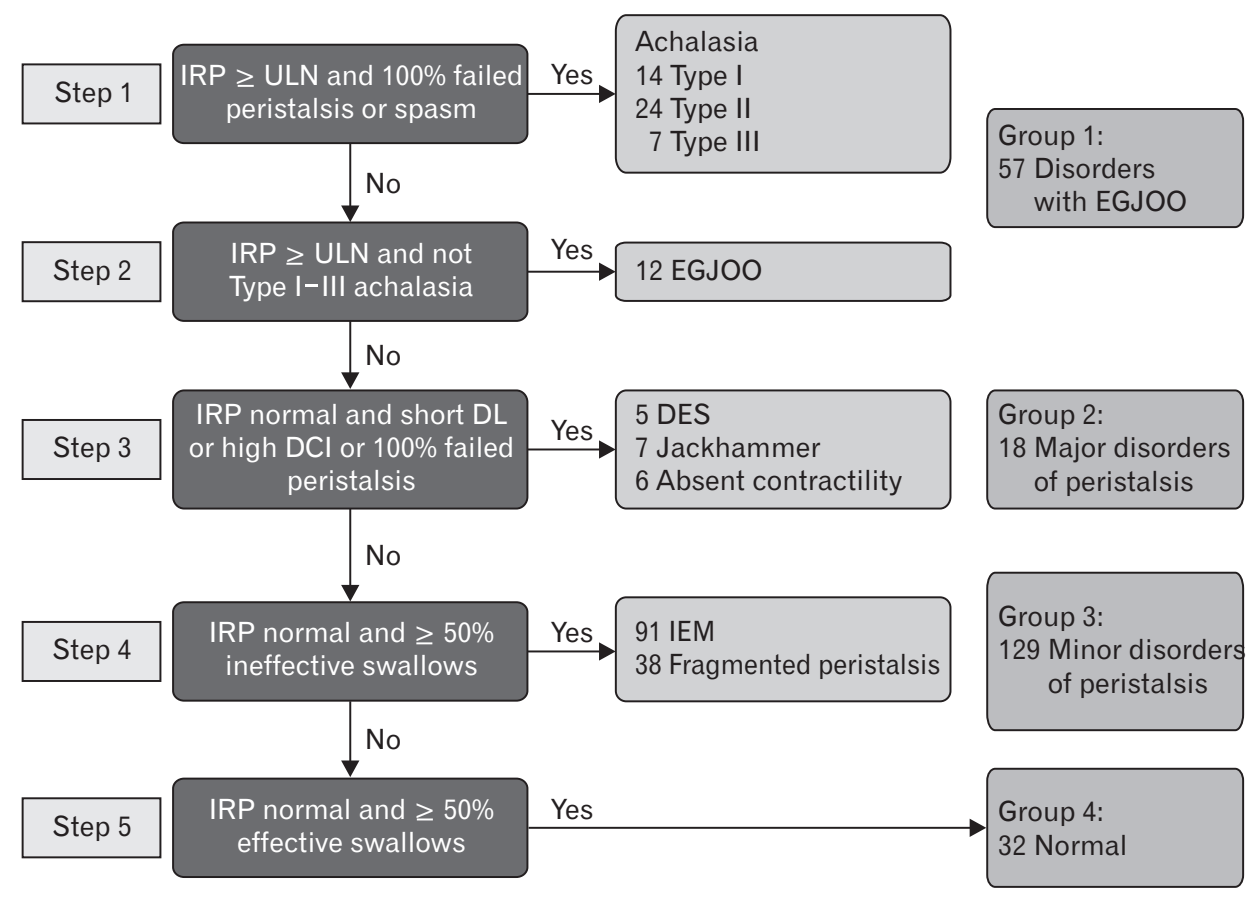

Figure 1. Steps in classification according to hierarchical algorithm for the interpretation of high-resolution manometry studies with Chicago classification version 3.0. IRP, integrated relaxation pressure; ULN, upper limit of normal; EGJOO, esophagogastric junction outfow obstruction; DL, distal latency; DCI, distal contractile integral; DES, distal esophageal spasm; IEM, ineffective esophageal motility.

score. ${ }^{8,9}$ The main categories included dysphagia, regurgitation, retrosternal pain, and weight loss. Each item was scored on a scale of 0 to 3 (score 0 , not present; score 1, occasionally; score 2, daily; and score 3 , several times a day after each meal). The degree of weight loss was scored as follows: score 0 , none; score $1,<5 \mathrm{~kg}$; score 2, 5-10 kg; and score 3, > $10 \mathrm{~kg}$ ). The maximum score was 12 points; the greater the score, the greater was the severity of symptoms.

\section{Statistical Methods}

Continuous variables are reported as mean \pm SEM. Categorical data are reported as frequencies and proportions. Age and gender were compared between the 8 groups using non-parametric independent-samples Kruskal-Wallis and Pearson's chi-square tests, respectively. One-way ANOVA was used for Eckardt score within the different motor disorders groups and to compare these groups with the normal esophageal manometry group. All statistical analyses were performed using statistical software package SPSS version 17.0 (IBM Corp, Armonk, NY, USA). In all cases, $P<0.05$ was required for statistical significance.

\section{Results}

\section{Demographic and Clinical Characteristics}

A total of 256 patients with esophageal dysphagia who exhibited normal esophageal appearance on EGD were identified during the study reference period (2011-2015). Of these, 17 patients who were diagnosed with $\mathrm{HH}$ based on HRM and barium examination, and 3 patients who were diagnosed with systemic sclerosis (scleroderma) were excluded. Finally, 236 patients (90 male, 146 female) with a mean age of $48.4 \pm 12.2$ years (range, 18-85) were included in the analysis.

\section{High-resolution Manometry Classification}

Of 236 patients, 204 (86.4\%) patients had abnormal esophageal motility according to the CC v3.0. The HRM classification of motor disorders is described in Table and Figure 2. Achalasia was diagnosed in 45 (19.1\%) patients (type I: 14 [31.1\%, 14/45]; type II: 24 [53.3\%, 24/45], and type III: 7 [15.6\%, 7/45]). EGJOO was diagnosed in $12(5.1 \%)$ patients. In the major disorders group, $6(2.5 \%)$ patients were diagnosed with lack of contractility, 5 (2.1\%) patients with DES, and 7 (3.0\%) patients with jackhammer esophagus. In the minor disorders group, 91 (38.6\%) patients were diagnosed with IEM, and 38 (16.1\%) patients were diagnosed with 

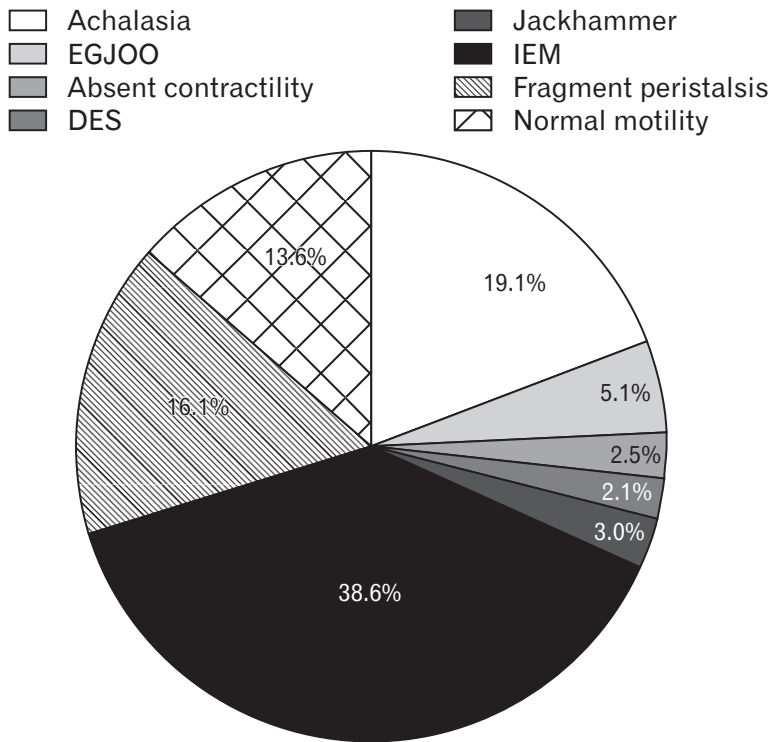

Figure 2. High-resolution manometry classification according to Chicago classification version 3.0. Of the 236 patients, categorical data are reported using proportions: ineffective esophageal motility (IEM) $38.6 \%$ (91/236), achalasia $19.1 \%$ (45/236), fragmented peristalsis $16.1 \%$ (38/236), esophagogastric junction outflow obstruction (EGJOO) $5.1 \%$ (12/236), Jackhammer esophagus 3.0\% (7/236), absent contractility $2.5 \%$ (6/236), and distal esophageal spasm (DES) $2.1 \%$ (5/236). Normal esophageal motility group $13.6 \%$ (32/236).

fragmented peristalsis.

Thirty-two (13.6\%) patients were classified as having normal esophageal manometry. Among these, 27 patients were diagnosed with esophageal motor disorders classified according to the previous CC v2.0 criteria. Of these 27 patients, 3 patients were diagnosed with jackhammer esophagus with only one with swallowing distal contractile integral over $8000 \mathrm{mmHg} \cdot \mathrm{sec} \cdot \mathrm{cm} ; 17$ patients had "nutcracker" esophagus (this designation was eliminated in CC v3.0), 2 patients had weak peristalsis with large peristaltic defects, 3 patients had weak peristalsis with small peristaltic defects, and 2 patients had frequent failed peristalsis. As these "motor disorders" patients did not fulfill the CC v3.0, they were included in the normal esophageal manometry group. However, only 5 patients were classified as having normal motility according to both criteria.

There were 44 NERD patients (GERD questionnaire positive) all of whom were classified in the minor disorders group (IEM $=34$, fragmented peristalsis $=10$ ). All of these patients underwent 24-hour $\mathrm{pH}$ monitoring or impendence monitoring, of which only 23 patients (IEM $=21$, fragmented peristalsis $=2)$ tested positive. 


\section{Questionnaire}

According to motor category, the Eckardt score was $4.7 \pm 0.1$ with esophageal outflow obstruction (achalasia: $4.8 \pm 0.1$, EGJOO: $4.1 \pm 0.3), 4.5 \pm 0.3$ with major motor disorders (DES: $4.6 \pm 0.5$, jackhammer esophagus: $4.7 \pm 0.4$, absent contractility: $4.2 \pm 0.5$ ), $3.5 \pm 0.1$ with minor motor disorders (IEM: $3.3 \pm$ 0.1 , fragmented peristalsis: $3.8 \pm 0.1$ ), and $3.9 \pm 0.1$ with normal esophageal manometry. There was significant difference between the Eckardt scores of patients with achalasia (4.8 \pm 0.1$)$, type I (4.6 $\pm 0.1)$, type II (5.0 \pm 0.2$)$, type III ( $4.7 \pm 0.3)$, and jackhammer esophagus $(4.7 \pm 0.4)$, and those of patients in the normal esophageal manometry group $(3.9 \pm 0.1)(P<0.05$; Table). There was also no significant difference between the scores of patients with EGJOO, DES, absent contractility, or fragmented peristalsis and those in the normal esophageal manometry group. However, the IEM patients scored significantly lower $(3.3 \pm 0.1)$ than those in the normal esophageal manometry group and others $(P<0.05)$.

\section{Discussion}

In this study, $86.4 \%$ of patients had esophageal motor disorders. Based on the CC v3.0, IEM was the most common esophageal motor disorder in our subjects, followed by achalasia, fragmented peristalsis, EGJOO, jackhammer esophagus, absent contractility, and DES. However, the severity of symptoms was related to the classification of esophageal motor disorders; a high Eckardt score suggested outflow obstruction or a major motor disorder, while a low score suggested IEM.
Classification of esophageal dysphagia in Chinese patients is not well characterized. Moreover, previous studies have shown significant variability in this respect. In our study, IEM was the most common esophageal motor disorder, followed by achalasia. In a study by Burgess and Wyeth, ${ }^{10}$ the most common motor disorder was achalasia followed by IEM. In another study of 403 patients with dysphagia, 53\% patients had normal motility. ${ }^{11}$ Esophageal motor disorders have been categorized into different patterns by different research groups, which might be attributable to differences with respect to the testing equipment, sample sizes and, possibly, to the ambiguous and overlapping classification criteria. The diagnostic criteria for esophageal motor disorders based on HRM should supersede the previous classification criteria. Development of clear definitions and standardized criteria based on esophageal manometry will help resolve the problem.

Esophageal dysphagia is classified into 2 categories based on the results of EGD and biopsies: obstructive and non-obstructive (Fig. 3). ${ }^{1}$ Obstructive esophageal dysphagia is caused by malignant strictures, benign strictures, and eosinophilic esophagitis or as a complication of surgery. Non-obstructive etiology may be classified as esophageal motor disorders and functional dysphagia based on the detection of motility disorders with manometry. In addition, non-obstructive etiology may also be classified as primary motor disorders (achalasia, DES, and jackhammer esophagus), secondary motor disorders (such as in patients with diabetes and scleroderma), and non-specific esophageal motor disorders. Use of these different diagnostic algorithms may cause confusion in clinical settings. Classification of NOD based on motility patterns using the HRM classification criteria is a simple and consistent method that yields

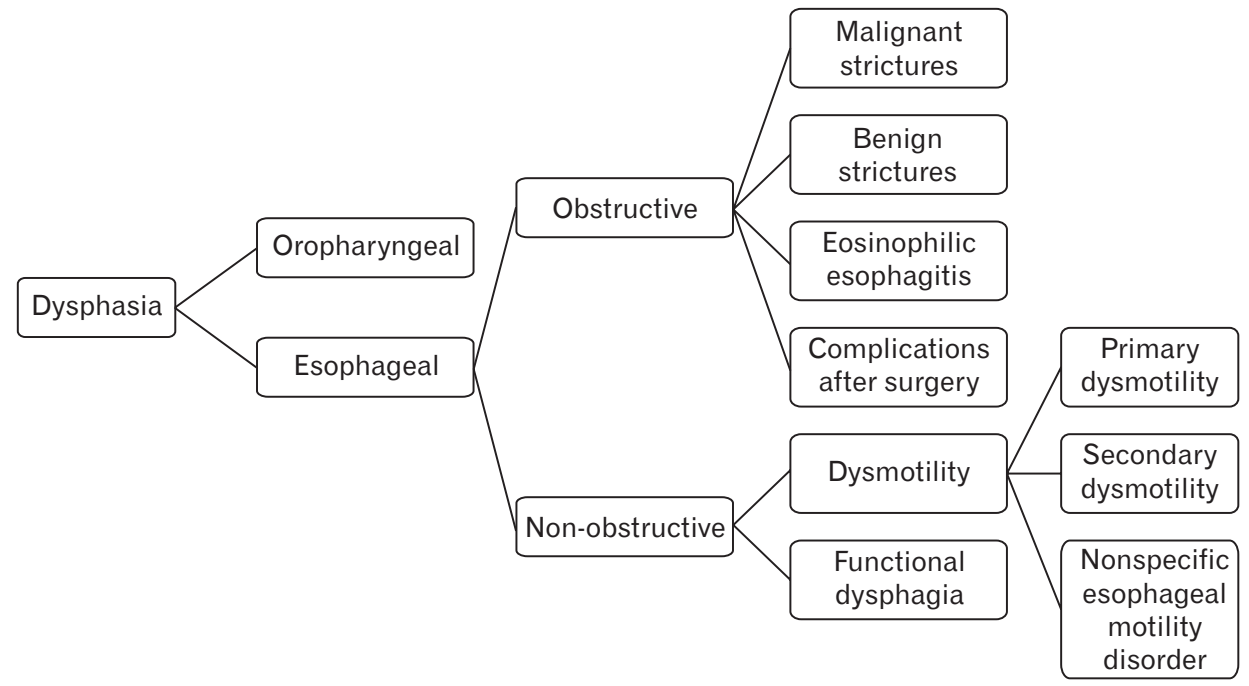

Figure 3. Classification of esophageal dysphagia based on potential underlying mechanism or etiology. 
4 categories: achalasia and EGJOO, major disorders of peristalsis, minor disorders of peristalsis, and normal esophageal manometry (Fig. 1). HRM is now considered the gold-standard for evaluation of esophageal motor disorders. Furthermore, it allows classification of all patients, although the spectrum of non-obstructive etiology is much broader than that of esophageal motor disorders. In our study, 32 patients with normal esophageal manometry could not be classified as having motor disorders or functional dysphagia, but rather as having non-obstructive esophageal dysphagia with normal manometry. Considering this advantage of HRM classification, we used it to classify esophageal dysphagia patients based on motility types.

In this study, normal HRM findings were observed in some patients with esophageal dysphagia. However, we could not rule out esophageal motor disorders based on a single normal esophageal manometry. Firstly, different classification criteria would define different "normal" manometry patients. In our normal esophageal manometry group, only 5 of 32 patients had normal manometry according to both 2012 and 2015 Chicago classification criteria. It was difficult to define whether the other 27 dysphagia patients had truly normal motility. They were thus classified as having 'normal' motility, since we currently have no evidence of specific esophageal motor disorders. However, we still think that the 17 patients who previously were diagnosed with nutcracker esophagus and 3 patients who were diagnosed with jackhammer esophagus were most likely to have esophageal motor disorders, even though they were not classified as such by the 2015 CC v3.0. "Normal esophageal manometry" might be a more appropriate expression than "normal esophageal motility.' The new classification criteria may falsely expand the number of patients labelled as having 'normal esophageal motility, and this will require application of new techniques to find evidence of motor disorders. Some studies suggest that related techniques such as functional luminal imaging probe (FLIP), videoscopy, or impedance planimetry of the esophagus may help detect subtle mechanical motor disorders in patients with dysphagia. ${ }^{12}$ Secondly, functional dysphagia might be included in the normal esophageal manometry group. The definition of functional dysphagia emphasizes the lack of evidence of structural, mucosal or motor mechanisms, especially histopathology-based esophageal motor disorders; ${ }^{13}$ however, the results of esophageal manometry have not been specified clearly. Therefore, the normal esophageal manometry group may include patients who have functional dysphagia after excluding major esophageal motor disorders. A final possible reason that should not be ignored is the somatization disorder. Psychological assessment should be completed before and after treatment in order to rule out possible psychological or emotional factors. We plan to incorporate this in our future studies. Nevertheless, normal esophageal manometry findings suggest that motor disorders might not be the unique cause of the symptoms.

Some limitations of our study should be highlighted. Firstly, conventional biopsy was not performed from all patients with normal mucosal appearance on EGD. Usually, EGD for evaluation of esophageal reflux symptoms in Western countries includes conventional biopsy from the lower esophagus to rule out eosinophilic esophagitis; however, the yield is rather low, ${ }^{14}$ and the sensitivity of detecting basal cell hyperplasia is also low. None of the established histological parameters can fulfill standards of a diagnostic tool. ${ }^{15,16}$ Thus, routine biopsy is not recommended in patients who do not exhibit visible esophageal erosions in China. ${ }^{17}$ However, the need for routine biopsy in such patients in the Chinese context should be evaluated in large-scale studies. Additionally, we did not use FLIP or esophageal impedance planimetry, as these techniques were not widely available at the time of the study.

In conclusion, IEM was the most common esophageal motor disorder in our patients with esophageal dysphagia who had normal EGD. In addition, the symptoms were associated with the classification of the esophageal motor disorders; a high Eckardt score suggests outflow obstruction or a major motor disorder, while a low score suggests IEM. Finally, NERD can be associated with IEM in dysphagia patients. It will be necessary to incorporate psychological assessment and strengthen the follow-up in future studies. In the meantime, continuously improving and perfecting the Chicago classification of the esophageal HRM should also be a priority.

Acknowledgement: Poster presentation at the Annual Meeting of the American Gastroenterological Association San Diego, May 2016.

Financial support: This study was partially funded through the JiLin Province Department of Finance (Grant No. 2016swszx026).

\section{Conflicts of interest: None.}

Author contributions: Dan Wang: study concept and design, data analysis, manuscript preparation, critical review, and final approval of manuscript; Xiu Wang, Yao Yu, Xiaowen Xu, Jing Wang, and Yuting Jia: data collection and analysis, and manuscript preparation; and Hong $\mathrm{Xu}$ : critical review and final approval of manuscript. 


\section{References}

1. Zerbib F, Omari T. Oesophageal dysphagia: manifestations and diagnosis. Nat Rev Gastroenterol Hepatol 2015;12:322-331.

2. Naini P, Dutta SK, Karhadkar AS, et al. Critical evaluation of esophageal dilation in nonobstructive dysphagia with and without esophageal rings. J Clin Gastroenterol 2007;41:362-365.

3. Elvevi A, Bravi I, Mauro A, et al. Effect of cold water on esophageal motility in patients with achalasia and non-obstructive dysphagia: a highresolution manometry study. J Neurogastroenterol Motil 2014;20:79-86.

4. Gyawali CP, Bredenoord AJ, Conklin JL, et al. Evaluation of esophageal motor function in clinical practice. Neurogastroenterol Motil 2013;25:99133.

5. Gor P, Li Y, Munigala S, Patel A, Bolkhir A, Gyawali CP. Interrogation of esophagogastric junction barrier function using the esophagogastric junction contractile integral: an observational cohort study. Dis Esophagus 2016;29:820-828.

6. Kahrilas PJ, Bredenoord AJ, Fox M, et al. The Chicago classification of esophageal motility disorders, v3.0. Neurogastroenterol Motil 2015;27:160-174.

7. Bredenoord AJ, Fox M, Kahrilas PJ, et al. Chicago classification criteria of esophageal motility disorders defined in high resolution esophageal pressure topography. Neurogastroenterol Motil 2012;24(suppl 1):57-65.

8. Eckardt VF, Aignherr C, Bernhard G. Predictors of outcome in patients with achalasia treated by pneumatic dilation. Gastroenterology
1992;103:1732-1738.

9. Eckardt AJ, Eckardt VF. Treatment and surveillance strategies in achalasia: an update. Nat Rev Gastroenterol Hepatol 2011;8:311-319.

10. Burgess NG, Wyeth JW. An audit of combined multichannel intraluminal impedance manometry in the assessment of dysphagia. J Gastroenterol Hepatol 2011;26(suppl 3):79-82.

11. Dekel R, Pearson T, Wendel C, De Garmo P, Fennerty MB, Fass R. Assessment of oesophageal motor function in patients with dysphagia or chest pain - the clinical outcomes research Initiative experience. Aliment Pharmacol Ther 2003;18:1083-1089.

12. Lin Z, Nicodème F, Boris L, Lin CY, Kahrilas PJ, Pandolfino JE. Regional variation in distal esophagus distensibility assessed using the functional luminal imaging probe (FLIP). Neurogastroenterol Motil 2013;25:e765-e771.

13. Galmiche JP, Clouse RE, Bálint A, et al. Functional esophageal disorders. Gastroenterology 2006;130:1459-1465.

14. Takubo K, Honma N, Aryal G, et al. Is there a set of histologic changes that are invariably reflux associated? Arch Pathol Lab Med 2005;129:159-163.

15. Zhou LY, Wang Y, Lu JJ, et al. Accuracy of diagnosing gastroesophageal reflux disease by GerdQ, esophageal impedance monitoring and histology. J Dig Dis 2014;15:230-238.

16. Schindlbeck NE, Wiebecke B, Klauser AG, Voderholzer WA, MullerLissner SA. Diagnostic value of histology in non-erosive gastro-oesophageal reflux disease. Gut 1996;39:151-154.

17. Chinese society of Gastroenterology. [2014 Chinese consensus of gastroesophageal reflux disease.] Chin J Dig 2014;34:649-661. [Chinese] 